 \\ TATRA MOUNTaINS

\section{ON THE SHARKOVSKY'S PROPERTY OF DARBOUX FUNCTIONS}

\author{
RYsZARD J. PAWLAK
}

\begin{abstract}
Examinations of Sharkovsky's property for discontinuous functions are usually connected with complex considerations. In this paper we ascertain the relation between two families of functions giving possibility to resolve the question about Sharkovsky's property of functions belonging to one of those families into examination $\mathcal{J}_{1}$ property of the second of them.
\end{abstract}

\section{Introduction}

On the website http://at.yorku.ca/t/a/i/c/41.htm, M. M is i u rewicz has written: Combinatorial Dynamics has its roots in Sharkovsky's Theorem 1 . Consequently, it seems to be important to consider Sharkovsky's property also for discontinuous functions $([6],[8])$. It is well known that continuous functions and functions possessing connected and $G_{\delta}$ graphs are Sharkovsky's functions (e.g., [1], 8]). However, there exist functions which have Sharkovsky's property and do not fulfil the above assumptions (for example, some functions belonging to families $\mathcal{P}_{\mathcal{C}}$ and $\mathcal{P}_{\mathcal{D}}$ defined in the second part of this paper). However, a question when a fixed function possesses Sharkovsky's property is rather difficult. So, it seems to be interesting to give a method permitting to replace the investigation of Sharkovsky's property of a given functions (or functions belonging to some family of functions) with applying results connected with property $\mathcal{J}_{1}$ of the wellknown classes of functions (in fact, Sharkovsky's property is closely connected with the property $\mathcal{J}_{1}$ also for a well-known class of functions, e.g., [8]). In order to attain it, we will establish some relation between functions (more precisely between the class of functions containing considered one) and suitable family

2000 Mathematics Subject Classification: 26A18, 26A15, 37E15, 54C40, 54H25, $26 \mathrm{~A} 15$.

Keywords: Darboux function, periodic point, Sharkovsky's function, property $\mathcal{J}$, family substituted by a class of functions.

${ }^{1}$ The name Sharkovsky, which appears in many English written papers, is spelled differently. I would like to use Misiurewicz's spelling from the web page. 
of functions possessing property $\mathcal{J}_{1}$, which will give us a possibility to infer Sharkovsky's property of considered function.

\section{Preliminaries}

We will use standard definitions and notations mostly (see [1, [2], [5]). In particular, by $\mathbb{N}, \mathbb{R}$ we will denote the set of positive integers, real numbers.

The interior of a set $A$ will be denoted by $\operatorname{Int}(A)$.

If $A, B$ are subsets of the domain of $f$, then $f \uparrow A$ denotes the restriction of $f$ to $A$. We say that a set $A f$-covers a set $B$ (denoted by $A \underset{f}{\longrightarrow} B$ ) if $B \subset f(A)$.

Let $f$ be a function. A point $x$ such that $f^{M}(x)=x$, but $f^{n}(x) \neq x$, for $n \in\{1,2, \ldots, M-1\}$ is called a periodic point of $f$ of prime period $M$. The set of all periodic points of $f$ of prime period $M$ will be denoted by $\operatorname{Per}_{M}(f)$.

If $\mathcal{F}$ is a family of functions mapping $\mathbb{R}$ into $\mathbb{R}$, then we shall denote

$$
\mathcal{F}^{c}=\left\{f_{n} \circ f_{n-1} \circ \cdots \circ f_{1}: f_{1}, f_{2}, \ldots, f_{n} \in \mathcal{F}, n \geq 1\right\} .
$$

Let $\mathcal{D}\left(\mathcal{B}_{1}, \mathcal{C}\right)$ denote the class of all Darboux functions, i.e., functions having Darboux property or, in other words, intermediate value property ([3]) (functions in Baire class 1, continuous functions). If we wish to consider the intersection of two classes, we shall write them next to each other, e.g., $\mathcal{D} B_{1}$ consists of all Darboux functions in Baire class 1.

Now, we can consider the following Sharkovsky's ordering of the set of all positive integers.

$$
\begin{aligned}
3 \prec 5 \prec 7 \prec \cdots \prec 2 \cdot 3 \prec 2 \cdot 5 \prec 2 \cdot 7 \prec \cdots \prec 2^{2} \cdot 3 \prec 2^{2} \cdot 5 \prec \ldots \\
\cdots \prec 2^{3} \prec 2^{2} \prec 2 \prec 2^{0}=1 .
\end{aligned}
$$

We shall say that $f$ is a Sharkovsky's function (or $f$ possesses Sharkovsky's property) if $\operatorname{Per}_{M}(f) \neq \emptyset$ and $M \prec N$, then $\operatorname{Per}_{N}(f) \neq \emptyset$.

In the next definitions, the addition of index numbers means addition modulo $M$ (i.e., $M+1=1)$. Let $\left(I_{1}, I_{2}, \ldots, I_{M}\right)$ be a finite sequence of continuums $\left(I_{i} \subset \mathbb{R}\right.$, for $\left.i=1,2, \ldots, M\right)$ and let $f_{1}, f_{2}, \ldots, f_{M}: \mathbb{R} \longrightarrow \mathbb{R}$. We say that $\left(I_{1}, I_{2}, \ldots, I_{M}\right)$ is $\left(f_{1}, f_{2}, \ldots, f_{M}\right)$-cycle if

$$
I_{1} \underset{f_{1}}{\longrightarrow} I_{2} \underset{f_{2}}{\longrightarrow} I_{3} \underset{f_{3}}{\longrightarrow} \ldots \underset{f_{M-1}}{\longrightarrow} I_{M} \underset{f_{M}}{\longrightarrow} I_{M+1}=I_{1} \text {. }
$$

If $f_{1}=f_{2}=\cdots=f_{M}=f$, we say that a $\left(f_{1}, f_{2}, \ldots, f_{M}\right)$-cycle $\left(I_{1}, I_{2}, \ldots, I_{M}\right)$ is $(f)$-cycle.

\footnotetext{
${ }^{2}$ In the whole paper, if we have a sequence consisting of $M$ elements, numbered from 1 to $M$, then we accept $M+1=1$ (the addition of index numbers is modulo $M$ ).
} 


\section{ON THE SHARKOVSKY'S PROPERTY OF DARBOUX FUNCTIONS}

If $x_{0} \in I_{1}$ is a point such that

$$
\left(f_{i} \circ f_{i-1} \circ \cdots \circ f_{1}\right)\left(x_{0}\right) \in I_{i+1} \quad \text { for } \quad i \in\{1,2, \ldots, M\},
$$

we say that $x_{0}$ is connected with an $\left(f_{1}, f_{2}, \ldots, f_{M}\right)$-cycle $\left(I_{1}, I_{2}, \ldots, I_{M}\right)$.

We say $([8])$ that a family of functions $\mathcal{F}$ has property $\mathcal{J}$ if for any

$$
\left(f_{1}, f_{2}, \ldots, f_{M}\right) \text {-cycle }\left(I_{1}, I_{2}, \ldots, I_{M}\right)\left(f_{1}, f_{2}, \ldots, f_{M} \in \mathcal{F}\right),
$$

there exists a point $x_{0}$ connected with this cycle such that $\left(f_{M} \circ f_{M-1} \circ \cdots \circ\right.$ $\left.f_{1}\right)\left(x_{0}\right)=x_{0}$.

We say that a family of functions $\mathcal{F}$ has the property $\mathcal{J}_{1}$ if for any $(f)$-cycle $\left(I_{1}, I_{2}, \ldots, I_{M}\right)(f \in \mathcal{F})$ there exists a point $x_{0}$ connected with this cycle and such that $f^{M}\left(x_{0}\right)=x_{0}$ (in the case of continuous functions defined in compact interval, the property $\mathcal{J}_{1}$ is connected with so-called Itinerary Lemma).

\section{Main results}

Throughout the paper we will assume that all considered functions are Darboux functions (and at the same time, all considered classes of functions consist of Darboux functions).

It is very useful to introduce the following notions.

We will say that an $(f)$-cycle $\left(J_{1}, J_{2}, \ldots, J_{M}\right)$ predominates $\left(f_{1}, f_{2}, \ldots, f_{M}\right)$-cycle, $\left(I_{1}, I_{2}, \ldots, I_{M}\right)$ if for each $i \in\{1,2, \ldots$ $\ldots, M\}$, there exists a homeomorphic embedding $\xi_{i}: J_{i} \rightarrow I_{i}$ such that

$$
\left(f_{i} \circ \cdots \circ f_{1}\right)\left(\xi_{1}(x)\right)=\xi_{i+1}\left(f^{i}(x)\right)
$$

for each point $x$ connected with $(f)$-cycle $\left(J_{1}, J_{2}, \ldots, J_{M}\right)$.

We say that a family of functions $\mathcal{F}$ is substituted by a class of functions $\mathcal{F}_{1}$ if for any natural number $M$ and any arbitrary $\left(f_{1}, f_{2}, \ldots, f_{M}\right)$-cycle $\left(I_{1}, I_{2}, \ldots, I_{M}\right)$, where $f_{1}, \ldots, f_{M} \in \mathcal{F}$, there exists an $(f)$-cycle $\left(J_{1}, J_{2}, \ldots, J_{M}\right)$ which predominates $\left(f_{1}, f_{2}, \ldots, f_{M}\right)$-cycle $\left(I_{1}, I_{2}, \ldots, I_{M}\right)$ such that $f \in \mathcal{F}_{1}$.

First, we shall establish two classes of functions $\mathcal{P}_{\mathcal{C}}$ and $\mathcal{P}_{\mathcal{D}}$, which will form a model for our considerations. Let us note that some functions belonging to $\mathcal{P}_{\mathcal{C}}\left(\mathcal{P}_{\mathcal{D}}\right)$ have been considered in a lot of papers and monographs (e.g., [3], 4]).

Let $P$ be an arbitrary Cantor-like set in $[0,1]$ (for simplicity of considerations we assume that $0,1 \in P$ ) and let $P^{\prime} \subset P$. Then we can distinguish some properties of functions $f_{P^{\prime}, P}: \mathbb{R} \longrightarrow \mathbb{R}$ which are connected with the sets $P^{\prime}$ and $P$.

1. $f_{P^{\prime}, P}(x)=0$ if $x \in P \backslash P^{\prime}$ and if $P^{\prime} \neq \emptyset$, then $f_{P^{\prime}, P}(x)=1$, if $x \in P^{\prime}$. 
2. $f_{P^{\prime}, P} \uparrow[a, b]$ is a continuous function and $f_{P^{\prime}, P}([a, b])=[0,1]$ for any component $(a, b)$ of $[0,1] \backslash P$.

2'. $f_{P^{\prime}, P} \uparrow(a, b)$ is a continuous function, $f_{P^{\prime}, P} \uparrow[a, b]$ is a Darboux function and $f_{P^{\prime}, P}([a, b])=[0,1]$, for any component $(a, b)$ of $[0,1] \backslash P$.

3. $f_{P^{\prime}, P}(x)=f_{P^{\prime}, P}(0)$, for $x<0$ and $f_{P^{\prime}, P}(x)=f_{P^{\prime}, P}(1)$, for $x>1$.

Let $\mathcal{P}_{\mathcal{C}}\left(\mathcal{P}_{\mathcal{D}}\right)$ be a family of all functions $f_{P^{\prime}, P}$ fulfilling conditions $\mathbf{1}, \mathbf{2}$ and $\mathbf{3}\left(\mathbf{1}, \mathbf{2}^{\prime}, \mathbf{3}\right)$ for all possible pairs of sets $\left(P^{\prime}, P\right)$.

Let us remark that $\mathcal{P}_{\mathcal{C}} \subset \mathcal{D}\left(\mathcal{P}_{\mathcal{D}} \subset \mathcal{D}\right)$ and, moreover, both classes contain nonmeasurable (in the Lebesgue sense) functions (if the measure of $P$ is positive and $P^{\prime}$ is a nonmeasurable set).

Now, we will show that:

The family $\mathcal{P}_{\mathcal{C}}$ is substituted by a family $\mathcal{C}$.

The family $\mathcal{P}_{\mathcal{D}}$ is substituted by a family $\mathcal{D} B_{1}$.

Let $\left(I_{1}, I_{2}, \ldots, I_{M}\right)$ be a fixed $\left(f_{1}, f_{2}, \ldots, f_{M}\right)$-cycle, i.e.,

$$
I_{1} \underset{f_{1}}{\longrightarrow} I_{2} \underset{f_{2}}{\longrightarrow} \ldots \underset{f_{M-1}}{\longrightarrow} I_{M} \underset{f_{M}}{\longrightarrow} I_{1},
$$

where $f_{i}=f_{P_{i}^{\prime}, P_{i}} \in \mathcal{P}_{\mathcal{C}}\left(f_{i}=f_{P_{i}^{\prime}, P_{i}} \in \mathcal{P}_{\mathcal{D}}\right)$ (for $\left.i=1,2, \ldots, M\right)$. Let us fix $i_{0} \in\{1, \ldots, M\}$. If $\operatorname{Int}\left(I_{i_{0}}\right)$ is a subset of a component of the complement of $P_{i_{0}}$ then we can put $\left(a_{i_{0}}, b_{i_{0}}\right)=\operatorname{Int}\left(I_{i_{0}}\right)$ (of course, it is sufficient to consider nondegenerate intervals). In the opposite case, there exists an component $\left(a_{i_{0}}, b_{i_{0}}\right)$ of $[0,1] \backslash P_{i_{0}}$ such that $\left[a_{i_{0}}, b_{i_{0}}\right] \subset \operatorname{Int}\left(I_{i_{0}}\right)$. Thus for any $i \in\{1,2, \ldots, M\}$, there exists an interval $\left(a_{i}, b_{i}\right) \subset I_{i}$ such that $\left[a_{i}, b_{i}\right] \underset{f_{i}}{\longrightarrow} f_{i}\left(I_{i}\right)$. So, we have

$$
f_{i}\left(\left[a_{i}, b_{i}\right]\right) \supset f_{i}\left(I_{i}\right) \supset I_{i+1} \supset\left[a_{i+1}, b_{i+1}\right] \quad \text { for } \quad i=1,2, \ldots, M .
$$

Therefore,

$$
\left[a_{1}, b_{1}\right] \underset{f_{1}}{\longrightarrow}\left[a_{2}, b_{2}\right] \underset{f_{2}}{\longrightarrow} \cdots \underset{f_{M-1}}{\longrightarrow}\left[a_{M}, b_{M}\right] \underset{f_{M}}{\longrightarrow}\left[a_{1}, b_{1}\right] .
$$

Let $\left\{\left[a_{i}^{\prime}, b_{i}^{\prime}\right]\right\}_{i=1}^{M}$ be a sequence of disjoint intervals such that $b_{i}^{\prime}-a_{i}^{\prime}=b_{i}-a_{i}$ and put $\delta_{i}=a_{i}^{\prime}-a_{i}$. Then $\left[a_{i}^{\prime}, b_{i}^{\prime}\right]=\left[a_{i}, b_{i}\right]+\delta_{i}(i=1,2, \ldots, M)$.

Let $\xi_{i}:\left[a_{i}^{\prime}, b_{i}^{\prime}\right] \rightarrow I_{i}$ be defined by the formula

$$
\xi_{i}(x)=x-\delta_{i}, \quad i=1,2, \ldots, M .
$$

Of course, $\xi_{i}$ is a homeomorphic embedding such that $\xi_{i}\left(\left[a_{i}^{\prime}, b_{i}^{\prime}\right]\right)=\left[a_{i}, b_{i}\right]$.

Let us define a function $f: \mathbb{R} \rightarrow \mathbb{R}$ in the following way:

$$
f(x)=f_{i}\left(\xi_{i}(x)\right)+\delta_{i+1} \quad \text { for } \quad x \in\left[a_{i}^{\prime}, b_{i}^{\prime}\right]
$$

$(i=1,2, \ldots, M)$ and $f$ is linear function in the closure of any component of the complement of $\bigcup_{i=1}^{M}\left[a_{i}^{\prime}, b_{i}^{\prime}\right]$. 


\section{ON THE SHARKOVSKY'S PROPERTY OF DARBOUX FUNCTIONS}

It is easy to see that $f \in \mathcal{C}$ if we consider the family $\mathcal{P}_{\mathcal{C}}$, or $f \in \mathcal{D} B_{1}$ if we consider the family $\mathcal{P}_{\mathcal{D}}$.

Now, we shall show that $\left(\left[a_{1}^{\prime}, b_{1}^{\prime}\right],\left[a_{2}^{\prime}, b_{2}^{\prime}\right], \ldots,\left[a_{M}^{\prime}, b_{M}^{\prime}\right]\right)$ is $(f)$-cycle, i.e.,

$$
\left[a_{1}^{\prime}, b_{1}^{\prime}\right] \underset{f}{\longrightarrow}\left[a_{2}^{\prime}, b_{2}^{\prime}\right] \underset{f}{\longrightarrow} \ldots \underset{f}{\longrightarrow}\left[a_{M}^{\prime}, b_{M}^{\prime}\right] \underset{f}{\longrightarrow}\left[a_{1}^{\prime}, b_{1}^{\prime}\right] .
$$

Let us fix $i_{0} \in\{1,2, \ldots, M\}$. Then

$$
\begin{aligned}
f\left(\left[a_{i_{0}}^{\prime}, b_{i_{0}}^{\prime}\right]\right)=f_{i_{0}}\left(\xi_{i_{0}}\left(\left[a_{i_{0}}^{\prime}, b_{i_{0}}^{\prime}\right]\right)\right)+\delta_{i_{0}+1} & \\
& \supset\left[a_{i_{0}+1}, b_{i_{0}+1}\right]+\delta_{i_{0}+1}=\left[a_{i_{0}+1}^{\prime}, b_{i_{0}+1}^{\prime}\right],
\end{aligned}
$$

which proves (2).

Let $x$ be an arbitrary point connected with $(f)$-cycle

It means that

$$
\left(\left[a_{1}^{\prime}, b_{1}^{\prime}\right],\left[a_{2}^{\prime}, b_{2}^{\prime}\right], \ldots \ldots,\left[a_{M}^{\prime}, b_{M}^{\prime}\right]\right)
$$

$$
x \in\left[a_{1}^{\prime}, b_{1}^{\prime}\right]
$$

and

$$
f(x) \in\left[a_{2}^{\prime}, b_{2}^{\prime}\right], f^{2}(x) \in\left[a_{3}^{\prime}, b_{3}^{\prime}\right], \ldots, f^{M-1}(x) \in\left[a_{M}^{\prime}, b_{M}^{\prime}\right], f^{M}(x) \in\left[a_{1}^{\prime}, b_{1}^{\prime}\right] .
$$

So, we have:

$$
\begin{aligned}
f_{1}\left(\xi_{1}(x)\right) & =\xi_{2}(f(x)), \\
f_{2}\left(f_{1}\left(\xi_{1}(x)\right)\right) & =f_{2}\left(\xi_{2}(f(x))\right)=\xi_{3}\left(f^{2}(x)\right) .
\end{aligned}
$$

Now, we suppose that $\left(f_{i-1} \circ \cdots \circ f_{1}\right)\left(\xi_{1}(x)\right)=\xi_{i}\left(f^{i-1}(x)\right)$ for $i \geq 2$. Then we shall prove that

$$
\left(f_{i} \circ f_{i-1} \circ \cdots \circ f_{1}\right)\left(\xi_{1}(x)\right)=\xi_{i+1}\left(f^{i}(x)\right) .
$$

We can calculate

$$
\left(f_{i} \circ\left(f_{i-1} \circ \cdots \circ f_{1}\right)\right)\left(\xi_{1}(x)\right)=f_{i}\left(\xi_{i}\left(f^{i-1}(x)\right)\right)=\xi_{i+1}\left(f^{i}(x)\right) .
$$

This completes the proof of (1).

Remark. One can consider various modifications of our models. For example, we can replace the condition $\mathbf{2}$ (and $\mathbf{2}^{\prime}$ ) with

$\mathbf{2}^{\prime \prime} . f \uparrow[a, b] \in \mathcal{D} B_{1}$ and $f([a, b])=[0,1]$, for any component of $[0,1] \backslash P$.

Then such a family is also substituted by $\mathcal{D} B_{1}$.

Moreover, the assumption 3 suggests that one can consider the functions mapping $[0,1]$ into itself.

The following lemma is the basic statement for further considerations. 
Lемма 2.1. If a family $\mathcal{F}$ is substituted by a class of functions $\mathcal{F}_{1}$ and the family $\mathcal{F}_{1}$ has property $\mathcal{J}_{1}$, then the family $\mathcal{F}^{c}$ has property $\mathcal{J}$.

Proof. Let $f_{1}, f_{2}, \ldots, f_{M} \in \mathcal{F}^{c}$ and let $\left(I_{1}, I_{2}, \ldots, I_{M}\right)$ be $\left(f_{1}, f_{2}, \ldots, f_{M}\right)$ -cycle. We can suppose that $f_{k}=h_{k, N(k)} \circ h_{k, N(k)-1} \circ \cdots \circ h_{k, 1}$, where $h_{k, i} \in \mathcal{F}$, $(i=1,2, \ldots, N(k))$ and $k \in\{1,2, \ldots, M\}$. Thus

$$
\begin{aligned}
f_{M} \circ \cdots \circ f_{1}=h_{M, N(M)} \circ & \cdots \circ h_{M, 1} \circ h_{M-1, N(M-1)} \circ \cdots \\
& \cdots \circ h_{M-1,1} \circ \cdots \circ h_{1, N(1)} \circ \cdots \circ h_{1,1} .
\end{aligned}
$$

Let us denote $I_{M, N(M)+1}=I_{M+1}=I_{1}=\left[a_{M, N(M)+1}, b_{M, N(M)+1}\right]$, where $a_{M, N(M)+1}=a_{1}, b_{M, N(M)+1}=b_{1}$. Remark that $h_{M, N(M)-1} \circ \cdots \circ h_{M, 1}\left(I_{M}\right)$ is a connected set and $I_{M+1}$ is a subset of a connected set

$$
f_{M}\left(I_{M}\right)=h_{M, N(M)}\left(h_{M, N(M)-1} \circ \cdots \circ h_{M, 1}\left(I_{M}\right)\right) .
$$

Thus, there exist points $a_{M, N(M)}, b_{M, N(M)} \in h_{M, N(M)-1} \circ \cdots \circ h_{M, 1}\left(I_{M}\right)$ such that

and

$$
h_{M, N(M)}\left(a_{M, N(M)}\right)=a_{M, N(M)+1}
$$

$$
h_{M, N(M)}\left(b_{M, N(M)}\right)=b_{M, N(M)+1} .
$$

Of course, it is possible that $b_{M, N(M)}<a_{M, N(M)}$, however, we can restrict our considerations to the case $a_{M, N(M)}<b_{M, N(M)}$ with no loss of generality. Let us put $I_{M, N(M)}=\left[a_{M, N(M)}, b_{M, N(M)}\right]$. Since $h_{M, N(M)}$ is a Darboux function, then $I_{M, N(M)} \underset{h_{M, N(M)}}{\longrightarrow} I_{M, N(M)+1}$.

Now, we apply the above reasoning again. Note that

$$
a_{M, N(M)}, b_{M, N(M)} \in h_{M, N(M)-1}\left(h_{M, N(M)-2} \circ \cdots \circ h_{M, 1}\left(I_{M}\right)\right)
$$

which means that there exist points $a_{M, N(M)-1}, b_{M, N(M)-1}$ in

$$
h_{M, N(M)-2} \circ \cdots \circ h_{M, 1}\left(I_{M}\right)
$$

such that

$$
h_{M, N(M)-1}\left(a_{M, N(M)-1}\right)=a_{M, N(M)}
$$

and

$$
h_{M, N(M)-1}\left(b_{M, N(M)-1}\right)=b_{M, N(M)} .
$$

Put $I_{M, N(M)-1}=\left[a_{M, N(M)-1}, b_{M, N(M)-1}\right]$. Since $h_{M, N(M)-1}$ is a Darboux function, then $I_{M, N(M)-1} \underset{h_{M, N(M)-1}}{\longrightarrow} I_{M, N(M)}$.

Repeating this procedure again and again we obtain two sequences of points:

$$
a_{M, N(M)+1}, a_{M, N(M)}, a_{M, N(M)-1}, \ldots, a_{M, 1}
$$




\section{ON THE SHARKOVSKY'S PROPERTY OF DARBOUX FUNCTIONS}

and

such that

$$
b_{M, N(M)+1}, b_{M, N(M)}, b_{M, N(M)-1}, \ldots, b_{M, 1}
$$

$$
h_{M, i}\left(a_{M, i}\right)=a_{M, i+1}, \quad h_{M, i}\left(b_{M, i}\right)=b_{M, i+1} \quad(i=1,2, \ldots, N(M)) .
$$

Simultaneously, we have a sequence of intervals

$$
I_{M, i}=\left[a_{M, i}, b_{M, i}\right] \quad(i=1,2, \ldots, N(M), N(M)+1)
$$

such that

$$
I_{M, 1} \underset{h_{M, 1}}{\longrightarrow} I_{M, 2} \underset{h_{M, 2}}{\longrightarrow}{ }_{h_{M, N(M)-1}}^{\longrightarrow} I_{M, N(M)} \underset{h_{M, N(M)}}{\longrightarrow} I_{M, N(M)+1}=I_{M+1} .
$$

It is easy to verify that $I_{M, 1} \subset I_{M}$.

We can repeat the above procedure for successive numbers $M-1, M-2, \ldots, 1$. Consequently, for any $k \in\{1,2, \ldots, M\}$ we obtain sequences of points

and

$$
a_{k, N(k)+1}, a_{k, N(k)}, a_{k, N(k)-1}, \ldots, a_{k, 1}
$$

$$
b_{k, N(k)+1}, b_{k, N(k)}, b_{k, N(k)-1}, \ldots, b_{M, 1}
$$

such that

$$
\begin{gathered}
h_{k, i}\left(a_{k, i}\right)=a_{k, i+1}, h_{k, i}\left(b_{k, i}\right)=b_{k, i+1} \quad(i=1,2, \ldots, N(k)) ; \\
I_{k, i}=\left[a_{k, i}, b_{k, i}\right] \quad(i=1,2, \ldots, N(k), N(k)+1)
\end{gathered}
$$

and

$$
\begin{aligned}
I_{k, 1} & \subset I_{k} & & (k=1,2, \ldots, M) ; \\
I_{k, N(k)+1} & \subset I_{k+1} & & (k=1,2, \ldots, M) ; \\
h_{k, i}\left(I_{k, i}\right) & \supset I_{k, i+1} & & (k=1,2, \ldots, M) .
\end{aligned}
$$

Let us denote $W=\sum_{i=1}^{M} N(i)$.

Now, we will introduce new enumerations of sequences

$$
\left\{h_{1,1}, \ldots, h_{1, N(1)}, \ldots, h_{M, N(M)}\right\} \quad \text { and } \quad\left\{I_{1,1}, \ldots, I_{1, N(1)}, \ldots, I_{M, N(M)+1}\right\}
$$

preserving the order:

$$
\begin{gathered}
h_{1}^{0}=h_{1,1}, h_{2}^{0}=h_{1,2}, \ldots, h_{N(1)}^{0}=h_{1, N(1)}, h_{N(1)+1}^{0}=h_{2,1}, \ldots \\
\ldots, h_{N(1)+N(2)-1}^{0}=h_{2, M}, \ldots, h_{W}^{0}=h_{M, N(M)}, \\
I_{1}^{0}=I_{1,1}, I_{2}^{0}=I_{1,2}, \ldots, I_{N(1)}^{0}=I_{1, N(1)}, I_{N(1)+1}^{0}=I_{1, N(1)+1}, \\
I_{N(1)+2}^{0}=I_{2,2}, \ldots, I_{W+1}^{0}=I_{M, N(M)+1}=I_{1} .
\end{gathered}
$$


Making some basic observation, we obtain

$$
I_{1}^{0} \underset{h_{1}^{0}}{\longrightarrow} I_{2}^{0} \underset{h_{2}^{0}}{\longrightarrow} \ldots \underset{h_{N(1)-1}^{0} \longrightarrow}{\longrightarrow} I_{N(1)}^{0} \underset{h_{N(1)}^{0}}{\longrightarrow} I_{N(1)+1}^{0} \underset{h_{N(1)+1}^{0} \longrightarrow}{\longrightarrow} \underset{h_{W-1}^{0} \longrightarrow}{\longrightarrow} I_{W}^{0} \underset{h_{W}^{0}}{\longrightarrow} I_{W+1}^{0} .
$$

This means that $\left(I_{1}^{0}, \ldots, I_{W}^{0}\right)$ form an $\left(h_{1}^{0}, \ldots, h_{W}^{0}\right)$-cycle. According to our assumption, there exists an $\left(f_{0}\right)$-cycle $\left(J_{1}, \ldots, J_{W}\right)$ predominating the above cycle, where $f_{0} \in \mathcal{F}_{1}$.

Let $y_{0} \in J_{1}$ be a point connected with an $\left(f_{0}\right)$-cycle $\left(J_{1}, \ldots, J_{W}\right)$ such that $f_{0}^{W}\left(y_{0}\right)=y_{0}$ and let $\xi_{i}: J_{i} \rightarrow I_{i}(i=1, \ldots, W)$ be a homeomorphic embedding such that

$$
\left(h_{i}^{0} \circ \cdots \circ h_{1}^{0}\right)\left(\xi_{1}\left(y_{0}\right)\right)=\xi_{i+1}\left(f_{0}^{i}\left(y_{0}\right)\right) \quad(i=1,2, \ldots, W) .
$$

Let us put $x_{0}=\xi_{1}\left(y_{0}\right) \in I_{1}$.

We shall prove that $x_{0}$ is a required point fulfilling the conditions defined in property $\mathcal{J}$ for an $\left(f_{1}, \ldots, f_{M}\right)$-cycle $\left(I_{1}, \ldots, I_{M}\right)$.

Let us fix a number $k_{0}$ belonging to $\{1,2, \ldots, M\}$. Let us put

$$
W_{0}=\sum_{i=1}^{k_{0}} N(i) \text {. }
$$

By (5) we can write

$$
\begin{aligned}
\left(f_{k_{0}} \circ \cdots \circ f_{1}\right)\left(x_{0}\right) & =\left(h_{W_{0}}^{0} \circ \cdots \circ h_{1}^{0}\right)\left(x_{0}\right) \\
& =\xi_{W_{0}+1}\left(f_{0}^{W_{0}}\left(y_{0}\right)\right) \in I_{W_{0}+1} \\
& =I_{k_{0}, N\left(k_{0}\right)+1} \subset I_{k_{0}+1},
\end{aligned}
$$

which proves that $x_{0}$ is a point connected with $\left(f_{1}, \ldots, f_{M}\right)$-cycle $\left(I_{1}, \ldots, I_{M}\right)$. To complete the proof it is sufficient to show that

$$
\left(f_{M} \circ \cdots \circ f_{1}\right)\left(x_{0}\right)=x_{0} .
$$

Now, using (5), we compute:

$$
\left(f_{M} \circ \cdots \circ f_{1}\right)\left(x_{0}\right)=\left(h_{W}^{0} \circ \cdots \circ h_{1}^{0}\right)\left(x_{0}\right)=\xi_{W+1}\left(f_{0}^{W}\left(y_{0}\right)\right)=\xi_{1}\left(y_{0}\right)=x_{0} .
$$

According to Lemma 2.1 and [8], Lemma 3.3, we have:

LeMma 2.2. If a family $\mathcal{F}$ is substituted by a family $\mathcal{F}_{1}$ and the family $\mathcal{F}_{1}$ has the property $\mathcal{J}_{1}$, then for each $f \in \mathcal{F}^{c}$ such that $\operatorname{Per}_{P}(f) \neq \emptyset$ we have:

(1) if $P>1$ and $P$ is odd, then for every $Q>P$, and for every even $Q$ and for $Q=1$ there exist closed intervals $I_{1}, \ldots, I_{Q}$ such that

$$
I_{1} \underset{f}{\longrightarrow} \ldots \underset{f}{\longrightarrow} I_{Q} \underset{f}{\longrightarrow} I_{1}
$$




\section{ON THE SHARKOVSKY'S PROPERTY OF DARBOUX FUNCTIONS}

and moreover, for every $x \in I_{1}$ such that $f^{Q}(x)=x$ and $f^{i}(x) \in I_{i+1}$ for $i=1, \ldots, Q-1, x \in \operatorname{Per}_{Q}(f)$;

(2) if $P>2$ and $P$ is even, then there exist closed intervals $I_{1}$ and $I_{2}$ such that $I_{1} \underset{f}{\longrightarrow} I_{1}$ and $I_{1} \underset{f}{\longrightarrow} I_{2} \underset{f}{\longrightarrow} I_{1}$ and, moreover, for every $x \in I_{1}$ such that $f^{2}(x)=x$ and $f(x) \in I_{2}, f(x) \neq x$;

(3) if $P=2$, then there exists a closed interval $I_{1}$ such that $I_{1} \underset{f}{\longrightarrow} I_{1}$.

TheOREM 2.3. Let us suppose that $\mathcal{F}$ is a family of functions substituted by a family $\mathcal{F}_{1}$ and the family $\mathcal{F}_{1}$ has the property $\mathcal{J}_{1}$. Then each function $f \in \mathcal{F}^{c}$ is Sharkovsky's function.

P r o of. According to Lemma 2.1, $\mathcal{F}^{c}$ has the property $\mathcal{J}$.

Let $f$ be a fixed function belonging to $\mathcal{F}^{c}$ for which there exists a point $x_{0}$ in $\operatorname{Per}_{M}(f)$. We shall show that $\operatorname{Per}_{N}(f) \neq \emptyset$ for any natural number $N$ such that $M \prec N$.

The proof will be divided into 4 steps (note that the case $M=2^{m} \cdot r, N=2^{n}$, and $n>m$ one can reduce to the undermentioned cases). Of course, we generally assume that $M \prec N$.

I. Either $M$ is an odd number and $N$ is a positive integer, or $M$ is an even number and $N \in\{1,2\}$.

II. $M=2^{m}$, where $m>1$.

III. $M=2^{m} \cdot r(r>1$ is an odd number, $m \geq 1)$ and $N=2^{n}$, where $n \leq m$.

IV. $M=2^{m} \cdot r(r>1$ is an odd number $m \geq 1)$ and $N=2^{n} \cdot s$, where $s>1$.

The proof in the case I follows immediately from Lemma 2.1 and Lemma 2.2 Now, we shall prove the remaining cases.

Proof of case II. Of course, $N=2^{n}$, where $m>n$. In view of case I, it is sufficient to consider $n>1$. Let us consider a function $\xi=f^{\frac{N}{2}} \in \mathcal{F}^{c}$. So, we have $\xi^{2^{m-n+1}}\left(x_{0}\right)=f^{M}\left(x_{0}\right)=x_{0}$.

Now, we will show that

$$
x_{0} \in \operatorname{Per}_{2^{m-n+1}}(\xi) .
$$

On the contrary, suppose that there exists a positive integer $k<2^{m-n+1}$ such that $\xi^{k}\left(x_{0}\right)=x_{0}$. Then $f^{k \cdot 2^{n-1}}\left(x_{0}\right)=x_{0}$ and $k \cdot 2^{n-1}<M$.

We obtain a contradiction with the assumption that $x_{0} \in \operatorname{Per}_{M}(f)$.

In view of case I, we can deduce that there exists a point $y_{0}$ in $\operatorname{Per}_{2}(\xi)$. It is not difficult to verify that $y_{0} \in \operatorname{Per}_{N}(f)$.

Proof of the case III. In this case we put $\xi=f^{\frac{M}{r}}=f^{2^{m}} \in \mathcal{F}^{c}$. So, we have $x_{0} \in \operatorname{Per}_{r}(\xi)$. Since $r$ is an odd number, then on account of case I, there exists 
$z_{0} \in \operatorname{Per}_{2}(\xi)$. Then $z_{0} \in \operatorname{Per}_{2^{m+1}}(f)$. According to case II, we have $\operatorname{Per}_{N}(f) \neq \emptyset$, where $N=2^{n}$ and $n \leq m$.

Proof of case IV. For the proof of this case we will show by induction on $m$ that:

If $f \in \mathcal{F}^{c}, M=2^{m} \cdot r(r>1$ is an odd number and $m \geq 1$ is a positive integer) and $\operatorname{Per}_{M}(f) \neq \emptyset$, then $\operatorname{Per}_{N}(f) \neq \emptyset$, for each $N=2^{n} \cdot s(s>1$ is an odd number) such that $M \prec N$.

The first step of the induction: $m=1$. Let $x_{0} \in \operatorname{Per}_{2 \cdot r}(f)$. Let $\mu=f^{2} \in \mathcal{F}^{c}$. It is not difficult to verify that

$$
x_{0} \in \operatorname{Per}_{r}(\mu) .
$$

If there exists an odd number $d>1$ such that $\operatorname{Per}_{d}(f) \neq \emptyset$, then (according to the case I.) $\operatorname{Per}_{N}(f) \neq \emptyset$ for any $N$ such that $d \prec N$. So, in our considerations (in this step of induction) we will assume that there is no odd number $d>1$ such that $\operatorname{Per}_{d}(f) \neq \emptyset$.

We will continue further considerations in two cases.

1) $n=1$. Then $r<s$. In view of (8) and case I there exists $y_{0} \in \operatorname{Per}_{s}(\mu)$. We will show that

$$
y_{0} \in \operatorname{Per}_{2 \cdot s}(f) .
$$

It is obvious that $f^{2 \cdot s}\left(y_{0}\right)=y_{0}$. On the contrary we suppose that $f^{k}\left(y_{0}\right)=$ $y_{0}$ and $k<2 \cdot s$. According to our assumption, $k$ is an even number. So let $k=2 \cdot w(w<s)$. Thus $y_{0}=\mu^{w}\left(y_{0}\right)$ which is impossible.

2) $n>1$. Note that $N=2 \cdot k_{0}\left(k_{0} \geq 1\right.$ is some positive integer) and moreover

$$
r \prec k_{0} \text {. }
$$

According to case I, there exists $p_{0} \in \operatorname{Per}_{k_{0}}(\mu)$. Obviously, $p_{0} \in \operatorname{Per}_{2 \cdot k_{0}}(f)=$ $\operatorname{Per}_{N}(f)$. This finishes the proof of the first step of this induction.

Let us suppose that (7) is true for a positive integer $m-1(m>1)$. We shall prove that (7) is also true for the number $m$.

So, let $f \in \mathcal{F}^{c}, x_{0} \in \operatorname{Per}_{2^{m} \cdot r}(f)$ for some odd number $r>1$ and $N=2^{n} \cdot s$ $\left(s>1\right.$ is an odd number such that $\left.2^{m} \cdot r \prec N\right)$. Let us put $\xi=f^{2} \in \mathcal{F}^{c}$. It is not difficult to verify that $x_{0} \in \operatorname{Per}_{2^{m-1} \cdot r}(\xi)$.

Note that $N$ is an even number, and so $N=2 \cdot n_{0}\left(n_{0}=2^{n-1} \cdot s, n-1 \geq 1\right)$. Let us notice that

$$
2^{m-1} \cdot r \prec n_{0}=2^{n-1} \cdot s .
$$

According to (9) and the inductive supposition, we can infer that there exists $t_{0}$ in $\operatorname{Per}_{n_{0}}(\xi)$. Obviously, $t_{0} \in \operatorname{Per}_{2 \cdot n_{0}}(f)=\operatorname{Per}_{N}(f)$. 


\section{ON THE SHARKOVSKY'S PROPERTY OF DARBOUX FUNCTIONS}

Remark. It is known ([7], [8] ) that families $\mathcal{C}$ and $\mathcal{D} B_{1}$ have property $\mathcal{J}_{1}$. So, as a simple consequence of this theorem and the considerations beginning this paper, we have: any function $f \in \mathcal{P}_{\mathcal{D}}$ is a Sharkovsky's function (and consequently, any function $f \in \mathcal{P}_{\mathcal{C}}$ is a Sharkovsky's function).

Problem. If $\mathcal{F}$ is some family of functions possessing the property $\mathcal{J}_{1}$ and $\mathcal{F}_{1}$ is substituted by $\mathcal{F}$, then (Theorem 2.3) $\mathcal{F}_{1}^{c}$ consists of Sharkovsky's functions and, moreover, (Lemma 2.1) $\mathcal{F}_{1}^{c}$ has the property $\mathcal{J}_{1}$. Then we can consider a family $\mathcal{F}_{2}$, which is substituted by $\mathcal{F}_{1}^{c}$ (if such a family there exists). This leads us to the following question:

Whether for any family $\mathcal{F}$ of Darboux functions possessing the property $\mathcal{J}_{1}$ there exists a family $\mathcal{F}_{1} \neq \mathcal{F}$ which is substituted by $\mathcal{F}$.

\section{REFERENCES}

[1] AlSEDÀ, L.-LliBRE, J.-MISIUREWICZ, M.: Combinatorial Dynamics and Entropy in Dimension One. Adv. Ser. Nonlinear Dynam., Vol. 5, World Scientific, Singapore, 1993.

[2] BLOCK, L. S.-COPPEL, W. A.: Dynamics in One Dimension. Lecture Notes in Math., Vol. 1513, Springer-Verlag, Berlin, 1992.

[3] BRUCKNER, A. M.: Differentiation of Real Functions. CRM Monogr. Ser., Vol. 5, AMS, Providence, RI, 1994.

[4] BRUCKNER, A. M.-CEDER, J. G.: Darboux continuity, Jahresber. Deutsch. Math.-Verein. 67 (1965), 93-117.

[5] ENGELKING, R.: General Topology. Monografie Matematyczne, Vol. 60, PWN-Polish Scientific Publishers, Warszawa, 1977.

[6] KELLUM, K. L.: Iterates of almost continuous functions and Sharkovskii's theorem, Real Anal. Exchange 14 (1989), 420-422.

[7] SZUCA, P.: Punkty Stałe Odwzorowań Typu Darboux. Doctoral Thesis, Gdańsk, 2003. (In Polish)

[8] SZUCA, P.: Sharkovskiù's theorem holds for some discontinuous functions, Fund. Math. 179 (2003), 27-41.

Faculty of Mathematics and Computer Science

Eódź University

Banacha 22

PL-90-238 Łódź

POLAND

The College of Computer Science

Rzgowska 17 a

PL-93-008 Eódź

POLAND

E-mail: rpawlak@math.uni.lodz.pl 